\title{
A pós-graduação e as perspectivas de pesquisa em Ciência da Informação no Brasil
}

\author{
Posgraduate studies and research perspectives in Library and Information Science in Brazil
}

\author{
José Augusto Chaves Guimarães y Pedro José Menezes \\ Departamento de Ciência da Informação, Universidade Estadual Paulista - UNESP, Marília - Brasil \\ guimajac@marilia.unesp.br
}

\begin{abstract}
Resumen
A área de Ciência da Informação no Brasil (envolvendo estudos relativos a Biblioteconomia, Arquivologia e Documentação) vem se consolidando academicamente no decorrer das últimas três décadas, notadamente a partir dos programas de pós-graduação stricto sensu (mestrado e doutorado) na área, bem como por um complexo e eficiente sistema governamental de apoio à investigação científica por meio de agências de fomento. Dessa forma, o país pôde construir sua base epistemológica a partir de uma simbiose e da abordagem crítica de distintas influências científicas externas (notadamente inglesas, norte-americanas, francesas espanholas) e de uma reflexão própria sobre aspectos de natureza sócio-econômico-cultural inerentes à realidade brasileira, o que tem contribuído para o diálogo internacional na área, em termos de reflexão teórica e metodológica e contribuído para a participação do Brasil em ações interinstitucionais em âmbito internacional. Desse modo, apresenta-se um panorama do sistema brasileiro de pós-graduação e de incentivo à investigação científica, com especial destaque para a realidade da área de Ciência da Informação, concluindo-se, por um lado, que é ainda insuficiente do número de cursos de mestrado e doutorado existentes face às crescentes demandas de capacitação docente dos cursos de graduação na área e, por outro, que o forte investimento governamental por meio de políticas públicas de apoio à pesquisa, bem como a existência da ANCIB, como espaço acadêmico para reflexão e interlocução da comunidade científica da área, sinalizam para uma efetiva consolidação acadêmica da área no país.
\end{abstract}

Palabras clave: Posgrados. Ciencia de la información. Capacitación docente. Investigación científica.

\section{Introdução}

A área de Ciência da Informação, no Brasil, aqui entendida sob uma concepção abrangente que envolve os estudos relativos à Biblioteconomia, à Arquivologia e à Documentação, vem se consolidando academicamente no decorrer das últimas três décadas, notadamente a partir dos programas de pós-graduação stricto sensu

\begin{abstract}
The Information Science area in Brazil (including Library, Archival and Documentation studies) has been suffering an academic consolidation during the last ten years, specially by means of the development of $\mathrm{PhD}$ and MSc programs, as well as by a complex and efficient governmental system of research supporting. In this sense, Brazil could build its epistemological background based on foreign influences (specially North American, English, French and Spanish ones), as well on a self-reflection about social, economical, and cultural aspects inherent to the Brazilian reality. Such a scientific landscape has allowed the country to taking part in most of the theoretical and methodological international discussions in the Information Science scenery. Considering those questions, it presents a landscape of Brazilian postgraduate and research support system in Information Science which makes possible to conclude, on one side, that there are not enough $\mathrm{PhD}$ and MSc programs to respond to the growing demands of the area (specially the needs for professors in Archival and Library Science Undergraduate courses) and, on the other side, that the strong governmental research support policies and the existence of scientific associations like ANCIB furnish a very unique environment for scientific research, academic exchange and, as a consequence, the advance of Information Science in Brazil.
\end{abstract}

Keywords: Postgraduate studies. Information science. Brazil. Scientific research.

(mestrado e doutorado) na área bem como por um complexo e eficiente sistema governamental de apoio à investigação científica, por meio de agências de fomento.

Nesse sentido, destaca-se o fato de o país haver construído a sua base epistemológica na área a partir de uma simbiose e abordagem crítica de distintas influências científicas externas (notadamente inglesas, norte-americanas, 
francesas espanholas) ao que se alia a reflexão sobre aspectos de natureza sócio-econômicocultural inerentes à realidade brasileira.

Tais elementos têm permitido um efetivo diálogo na área, em termos de reflexão teórica e metodológica, e contribuído para a participação do Brasil em ações interinstitucionais em âmbito internacional.

A visto disso, apresenta-se um panorama sobre o sistema brasileiro de pós-graduação e de incentivo à investigação científica, com especial destaque para a realidade da área de Ciência da Informação.

\section{O sistema brasileiro de pós-graduação}

O ensino de pós-graduação, no Brasil, apresenta-se, segundo a legislação do país, estruturado em dois universos: a Pós-Graduação lato sensu, enquanto modalidade de educação continuada que engloba os denominados cursos de Especialização, diretamente voltados ao aperfeiçoamento de profissionais para o mercado de trabalho, e a Pós-Graduação stricto sensu que abriga o Mestrado e o Doutorado acadêmico e, mais recentemente, duas novas modalidades: o Mestrado Interinstitucional e Mestrado Profissional.

Os cursos lato sensu, têm por características o caráter eventual e esporádico, de abordagem de partes específicas de um determinado campo do saber, possuindo natureza mais pragmática, por estarem mais diretamente vinculados às necessidades específicas do mercado de trabalho. Os concluintes com aproveitamento desses cursos fazem jus ao título de especialista, sem a exigência de uma dissertação ou tese com tema ou abordagem cientificamente inovadores. Desse modo, não se pode inserir o pós-graduação lato sensu no âmbito mais específico da capacitação docente ou científica mas, geralmente, no campo do aperfeiçoamento profissional.

Em linha bastante próxima do pós-graduação lato sensu tem-se os mestrados profissionais, que decorrem especificamente de uma demanda de formação de profissionais especializados e críticos, diretamente ligados ao mercado de trabalho. Dessa forma, os mestrados profissionais constituem forma híbrida que, por um lado, busca o aperfeiçoamento profissional típico dos cursos de especialização e, por outro, garantem a profundidade e o rigor científico dos mestrados acadêmicos sem, no entanto, se voltarem para a capacitação científica e docente.

Os cursos de mestrado e de doutorado acadêmicos, por sua vez, possuem a missão precípua de formar docentes e investigadores e, dessa forma, constituem as grandes matrizes de formação de recursos humanos especializados para os institutos de pesquisa e para o ensino universitário pois, como destaca Bertachini (1996, p. 46), essa modalidade de pósgraduação "busca reunir e integrar pesquisadores e docentes no desenvolvimento de conhecimentos". Dessa forma, para a autora, a capacitação docente

[...] deve se dar fundamentalmente no mestrado e no doutorado, espaço em que o processo reflexivo se desencadeia efetivamente, e no qual o indivíduo deve adquirir qualificações para a geração de conhecimento (através da pesquisa) e para a docência.

Esse aspecto se encontra claramente estabelecido nos termos do Lei de Diretrizes e Bases da Educação Nacional, no sentido do que a habilitação para o magistério superior se dá prioritariamente por meio de programas de mestrado e doutorado, ou seja no país, seja pelo reconhecimento de diplomas de mestrado e doutorado expedido por universidades estrangeiras. Indo além, o art. 52 da referida lei dispõe sobre a imprescindibilidade de que "um terço do corpo docente, pelo menos, com titulação acadêmica de mestrado e doutorado." (Brasil, 1996). Desse modo, é sobre a denominada pós-graduação stricto sensu (cursos de mestrado e doutorado) que se encontra assentada a formação de pesquisadores na área, uma vez que a carreira docente nas universidades públicas do país exige a concomitância de atividades de ensino e de pesquisa. Sob essa ótica, o mestrado interinstitucional, como destacam Smit, Dias e Souza $(2003$, p. 4) foi criado quando se percebeu que instituições de ensino superior tinham dificuldades em liberar seus professores para realizar mestrado em outras instituições. Assim, o Subprograma Mestrado Interinstitucional foi criado pela Coordenadoria de Aperfeiçoamento do Ensino Superior - CAPES, órgão do Governo Federal, com a sugestão que uma outra instituição promotora, com um programa de pósgraduação consolidado, ofereça-o no campus de uma outra instituição.

$\mathrm{Na}$ atualidade, pode-se dizer que o Brasil possui o maior, mais forte e sofisticado sistema de PósGraduação da América Latina, tendo expandido, no decorrer de 25 anos, $700 \%$ em programas de doutorado e $500 \%$ em cursos de mestrado (Morosini, 1999; Nunes, 1999).

O início formal dos estudos pós-graduados no Brasil remonta à década de 60 , quando Parecer 977/65 do Conselho Federal de Educação, definiu e distinguia as categorias de cursos lato e stricto sensu (Puterman, 2000, p.82). 
Em 1969, o Parecer 77 do Conselho Federal de Educação, art. 13, definiu o mestrado como etapa preliminar da pós-graduação, seguida do doutorado, como forma de "proporcionar formação científica ou cultural ampla e aprofundada, desenvolvendo a capacidade de pesquisa e poder criador nos diferentes ramos do saber" (Souza, 1991, p.133).

Em ambas as modalidades tem-se a frequência obrigatória a um conjunto de disciplinas e, ainda a elaboração e defesa pública de dissertação (no caso do mestrado, revelando aprofundamento científico) ou de tese (no caso do doutorado, revelando pleno ineditismo científico e inegável contribuição para o conhecimento do tema).

$\mathrm{Na}$ atualidade, a duração de um mestrado reside ao redor de 24 a 30 meses e, de doutorado, de 48 a 60 meses, exigindo-se a comprovação de proficiência em uma língua estrangeira para o mestrado e de duas para o doutorado. Em ambos os casos, tem-se a figura de um docente-orientador, portador de titulação mínima de doutor e devidamente credenciado no programa, que responde academicamente pela condução dos trabalhos de desenvolvimento de dissertação/tese.

Em termos de estrutura, os programas de pósgraduação se constroem a partir de linhas de pesquisa, caracterizadas por temáticas específicas que refletem o recorte epistemológico que a instituição de ensino faz sobre a área de estudo e que congregam grupos de docente/pesquisadores/orientadores.

Os cursos de pós-graduação stricto sensu, no Brasil, subordinam-se à coordenação e à supervisão da Coordenação de Aperfeiçoamento de Pessoal de Nível Superior - Capes, devendo ser por ela devidamente credenciados e, anualmente, alimentar o sistema DataCapes, onde figuram os relatórios de atividades dos cursos (que são públicos e disponíveis na página www.capes.gov.br), recebendo uma avaliação trienal que lhes garante uma pontuação entre 3 e 7 e, em casos de avaliação inferior, tem-se o descredenciamento do curso, com a perda de validade do título no país. A Capes é responsável também pelo reconhecimento ou não, no Brasil, de títulos de mestre e de doutor obtidos no exterior .

Outro aspecto extremamente significativo na atuação da Capes é o diretório Qualis, que avalia cientificamente todos os periódicos nos quais os docentes brasileiros publicam, classificandoos em internacionais, nacionais e regionais, (quanto à abrangência) e em $\mathrm{A}, \mathrm{B}$ e C (quanto à qualidade científica).
Aliado a esse sistema nacional de avaliação dos cursos de pós-graduação, desenvolvido pela Capes, tem-se o Currículo Lattes, a cargo do Conselho Nacional de Desenvolvimento Científico e Tecnológico - CNPq, no qual estão inscritas e constantemente atualizadas todas as informações curriculares dos docentes e pesquisadores brasileiros e também o Diretório de Grupos de Pesquisa, contendo as informações sobre as ações, a composição e os objetivos de todos os grupos de investigação existentes no país e devidamente credenciados para tal (informações disponíveis em www.cnpq.br).

Tais iniciativas visam a garantir transparência das informações relativas às ações empreendidas pelo ambiente científico brasileiro, e, especialmente no caso dos cursos de pós-graduação, à vida acadêmica dos docentes / pesquisadores, constituindo-se em importante informação pública e de livre acesso ao cidadão.

Desse modo, destaca Puterman (2000, p. 6566) que ao CNPq cabe promover e estimular o desenvolvimento da pesquisa científica e tecnológica em todos os domínios do conhecimento ao passo que a Capes tem por função aperfeiçoar o corpo docente das universidades e de instituições de ensino superior públicas, de modo a levar esses docentes a cumprirem sua missão de ensino e pesquisa científica, da maneira mais adequada.

Basicamente situados em instituições públicas, os cursos de pós-graduação brasileiros contam com forte investimento governamental por meio de bolsas de estudo, bolsas de pesquisa, equipamentos, material de pesquisa, etc, notadamente as partir de programas de apoio de fundações públicas de apoio à pesquisa tais como os já citados Capes e CNPq (ambos criados na década de 50), e ainda a Fundação de Financiamento de Estudos e Projetos - FINEP e as fundações estaduais de amparo à pesquisa, tais como FAPESP (São Paulo), FAPERGS (Rio Grande do Sul), FAPEMIG (Minas Gerais), FAPERJ (Rio de Janeiro), dentre outras. A isso se alia o financiamento proveniente de organismos internacionais, como o Banco Interamericano de desenvolvimento - BID (Martins, 2002, p.13).

\section{A pós-graduação em Ciência da Informação no Brasil}

No caso específico da Ciência da Informação (aqui englobando as áreas de Biblioteconomia, Arquivologia e Documentação), destaca-se, no contexto Latino-Americano, o fato de que "o Brasil possui estrutura própria de cursos de pósgraduação stricto sensu, fato que remonta aos 
primórdios da década de 70" (Guimarães, 2001, p. 5).

Especificamente no âmbito da Ciência da Informação, destacam Valentim e Guimarães (2002, p. 32) uma preocupação crescente quanto à capacitação do corpo docente na área de Ciência da Informação, como elemento para a garantia de qualidade das atividades de ensino e de pesquisa. Nesse contexto, a pesquisa, enquanto atividade científica desenvolvida nas universidades, atua como "ponto fundamental para o crescimento e maturidade docente $e$ discente" e reflete direta e positivamente no processo de ensino e aprendizagem e, conseqüentemente, na vida acadêmica dos alunos.

Tais aspectos ficaram claros, no contexto dos países do Mercosul, por ocasião do $\mathrm{V}$ Encontro de Diretores de Escolas de Biblioteconomia do Mercosul ocorrido em Assunção, em julho de 2001, quando, ao caracterizar a realidade docente da área, nos cinco países do grupo, assumiu-se a capacitação docente como uma condição fundamental para o desenvolvimento do ensino na área, com recomendações quanto ao estabelecimento de convênios nesse sentido.

O sistema brasileiro de pós-graduação na área de Ciência da Informação remonta ao início dos anos 70 e sofreu significativa influência teórica inglesa e norte-americana (a partir dos anos 70), francesa (a partir dos anos 80) e espanhola (a partir dos anos 90), uma vez que foram realizados fortes investimentos governamentais para que docentes se doutorassem no exterior.

Hoje, o país dispõe onze programas que oferecem estudos pós-graduados em nível de mestrado e sete em nível de doutorado na área de Ciência da Informação.

Os cursos de mestrado da área são oferecidos nas seguintes universidades: Pontifícia Universidade Católica de Campinas - PUCCAMP (Campinas - São Paulo); Universidade de Brasília - UnB (Brasília - Distrito Federal); Universidade Estadual Paulista - UNESP (Marília - São Paulo); Universidade Federal da Bahia - UFBA (Salvador - Bahia); Universidade Federal Fluminense - UFF (Niterói - Rio de Janeiro); Universidade Federal de Minas Gerais - UFMG (Belo Horizonte - Minas Gerais); Universidade Federal da Paraíba - UFPB (João Pessoa Paraíba); Universidade Federal de Santa Catarina - UFSC (Florianópolis - Santa Catarina); Universidade Federal do Rio Grande do Sul UFRGS (Porto Alegre - Rio Grande do Sul); UNIRIO - Universidade do Rio de Janeiro (Rio de Janeiro) e Universidade de São Paulo - USP (São Paulo).

\begin{tabular}{|c|c|c|c|}
\hline Instituição & Início & Nivel & Linhas de pesquisa \\
\hline PUCCAMP & 1977 & M & $\begin{array}{l}\text { Gestão de Serviços da } \\
\text { Informação } \\
\text { Produção e Disseminação da } \\
\text { Informação }\end{array}$ \\
\hline UnB & 1978 & M-D & $\begin{array}{l}\text { Gestão da informação e do } \\
\text { conhecimento } \\
\text { Arquitetura da informação } \\
\text { Comunicação da informação }\end{array}$ \\
\hline UNESP & 1998 & M-D & $\begin{array}{l}\text { Informação e tecnologia } \\
\text { Organização da informação }\end{array}$ \\
\hline UFBA & 1998 & M & $\begin{array}{l}\text { Informação e Contextos Sócio- } \\
\text { Econômicos } \\
\text { Teoria e Gestão do } \\
\text { Conhecimento }\end{array}$ \\
\hline UFF-IBICT & 1970 & $M-D$ & $\begin{array}{l}\text { Teoria, Epistemologia e } \\
\text { Interdisciplinaridade em Ciência } \\
\text { da Informação } \\
\text { Processamento e Tecnologia da } \\
\text { Informação } \\
\text { Configurações Sociais e Políticas } \\
\text { da Informação } \\
\text { Gestão da informação }\end{array}$ \\
\hline UFMG & 1976 & $M-D$ & $\begin{array}{l}\text { Informação, cultura e sociedade } \\
\text { Informação gerencial e } \\
\text { tecnológica } \\
\text { Organização e uso da } \\
\text { informação }\end{array}$ \\
\hline UFPB & 1978 & M & $\begin{array}{l}\text { Memória, Organização, } \\
\text { Produção e Uso da Informação }\end{array}$ \\
\hline UFSC & 2003 & $M$ & $\begin{array}{l}\text { Fluxo da informação } \\
\text { Profissional da informação }\end{array}$ \\
\hline UFRGS (1) & 1995 & $M-D$ & $\begin{array}{l}\text { Informação, Tecnologias e } \\
\text { Práticas Sociais } \\
\text { Comunicação, representações e } \\
\text { práticas culturais }\end{array}$ \\
\hline USP & 1972 & M-D & $\begin{array}{l}\text { Acesso à informação } \\
\text { Mediação e ação cultural }\end{array}$ \\
\hline UNIRIO (2) & 2005 & M-D & $\begin{array}{l}\text { Memória e Patrimônio } \\
\text { Memória e Espaço } \\
\text { Memória e Linguagem } \\
\text { Memória, Subjetividade e } \\
\text { Criação }\end{array}$ \\
\hline
\end{tabular}

Tabla I.

Os cursos de doutorado, por sua vez, são oferecidos na Universidade de Brasília, na Universidade Estadual Paulista, na Universidade Federal Fluminense; na Universidade Federal de Minas Gerais, na Universidade Federal do Rio Grande do Sul, na Universidade do Rio de Janeiro e na Universidade de São Paulo. Dessa forma, assim se coloca, na atualidade, o panorama geral dos cursos de pós-graduação em Ciência da Informação no Brasil (Tabla I). 
Analisando o panorama do ensino de pósgraduação em Ciência da Informação no Brasil, Smit, Dias e Souza (2002) e considerando as perspectivas de crescimento e as demandas da área no país (principalmente se consideramos a existência de cerca de 50 cursos de graduação em Biblioteconomia e Arquivologia), avaliam como ainda bastante tímido o número de cursos de mestrado e doutorado, e destacam a importância de esforços institucionais para a criação de novos cursos e em regiões diversificadas, uma vez que a grande maioria dos cursos de localiza no eixo centro-sul do país.

\section{A pesquisa em Ciência da Informação no Brasil}

As políticas e a agenda de pesquisa na área vem sendo objeto da atuação da Associação Nacional de Pesquisa e Pós-Graduação em Ciência da Informação - ANCIB (www.ancib. org.br), criada em 1989, que vem atuando significativamente como fórum de interlocução entre os cursos e entre os pesquisadores da área, notadamente por meio da realização dos Encontros Nacionais de Pesquisa e Pós-graduação em Ciência da Informação - ENANCIBs. Até o momento foram realizados sete ENANCIBs (Belo Horizonte, 1994; Valinhos, 1995; Rio de Janeiro, 1997; Brasília, 2000; Belo Horizonte, 2003; Florianópolis, 2005 e Marília, 2006), e o VIII ENANCIB realizar-se-á em Salvador, no final de outubro de 2007.

Os encontros da ANCIB atuam como espaços de interlocução acadêmica relativamente à produção de conhecimento em Ciência da Informação no Brasil e têm sua atividade pautada na atuação de sete grupos de trabalho (GTs) formalmente estruturados e que constituem, por tradição, os sub-temas de cada ENANCIB.

Contando com sete GTs, a ANCIB desenvolve suas ações de coordenação do universo investigativo em Ciência da Informação no Brasil a partir dos universos de conteúdo a seguir destacados:

O GT1, denominado Estudos Históricos e Epistemológicos da Informação propõe-se a investigar e discutir os "paradigmas da $\mathrm{Cl}$, a constituição do seu campo científico e as questões epistemológicas subjacentes", em cujo âmbito se incluem discussões sobre "disciplinaridade, interdisciplinaridade e transdisciplinaridade da área, bem como a construção do conhecimento em $\mathrm{Cl}$ do ponto de vista histórico".

As questões de organização e representação do conhecimento são objeto de estudo do GT2, mais especificamente no que se refere às "teorias, metodologias e práticas relacionadas à organização e preservação de documentos e da informação, enquanto conhecimento registrado e socializado", compreendendo a abordagem dos "processos, produtos e instrumentos de representação do conhecimento", as relações inter e transdisciplinares neles verificadas, e as questões de ligadas a "políticas de organização e preservação da memória institucional".

O GT-3, relacionado aos aspectos de Mediação, Circulação e Uso da Informação, busca investigar a relação da informação com os "processos culturais e simbólicos na contemporaneidade". Nesse âmbito, as discussões sobre redes sociais e redes que utilizam tecnologias, bem como formas de recepção em diferentes espaços e ambientes institucionais, aliam-se aos estudos sobre usos e usuários da informação e às temáticas de leitura, textualidade e memória.

A gestão da informação, de sistemas, de unidades, de serviços, de produtos e de recursos informacionais é o objeto do GT-4, denominado Gestão da Informação e do Conhecimento nas Organizações, que investiga especificamente os fluxos, processos e uso da informação na perspectiva da gestão, os estudos de usuários e a as questões de inteligência competitiva no contexto da Ciência da Informação. Para tanto, têm especial relevância os aspectos da gestão do conhecimento e da aprendizagem organizacional no contexto da Ciência da Informação, assim como da aplicabilidade das tecnologias da informação na gestão.

Os aspectos socioculturais, políticos, legais, éticos, econômicos e tecnológicos associados às transformações nos processos de geração,difusão/compartilhamento e uso da informação e do conhecimento inserem-se no âmbito investigativo do GT-5 -Política e Economia da Informação- em temas como: políticas de informação e suas expressões em ciência, tecnologia, saúde, meio ambiente, educação e cultura; as relações entre informação, Estado e governo, propriedade intelectual e acesso à informação e ao conhecimento; economia política da informação; informação, conhecimento e inovação; e inclusão informacional e digital.

O GT6, denominado Informação, Educação e Trabalho, busca investigar as relações entre informação, educação e trabalho na sociedade contemporânea, em temáticas relativas aos atores, cenários e estruturas que integram o campo de trabalho informacional assim como à formação e atuação do profissional de informação.

No âmbito da Produção e Comunicação da Informação em CT\&I, o GT7 investiga temáticas relativas a "medição, mapeamento, diagnóstico e avaliação da informação, nos processos de 
produção, armazenamento, comunicação e uso, em ciência, tecnologia, inovação e outros contextos". Para tanto, as análises quantitativas e qualitativas como a bibliometria, a cientometria, a infometria, a webmetria, e a análise de redes, assim como os indicadores em ciência, tecnologia e inovação encontram espaço de reflexão e discussão científica.

\section{Conclusão}

A pós-graduação e a pesquisa em Ciência da Informação, no Brasil, tiveram seu crescimento a partir da década de 70 , contando com um sólido sistema de investimentos públicos que vem contribuindo para a sedimentação teóricometodológica da área e para o desenvolvimento do ensino e da pesquisa por meio da formação de massa crítica.

Nesse contexto, as políticas públicas de credenciamento e avaliação contínua dos cursos de pós-graduação pela Capes, a publicidade dos currículos de todos os pesquisadores pelo CNPq e os investimentos em pesquisa (apoio a eventos, bolsas de pesquisa, apoio a infraestrutura de pesquisa, apoio a publicações, etc) têm permitido à área sua inserção nas agendas internacionais de pesquisa, bem como importantes diálogos interdisciplinares.

Relativamente às demandas de docentes/investigarores capacitados para o ensino de graduação na área, observa-se que a estrutura de pós-graduação é ainda tímida, mas com plenas capacidades de crescimento.

Por fim, destaca-se a consolidação da ANCIB como espaço acadêmico por excelência, onde os docentes e investigadores encontram condições para reflexão, interlocução e intercâmbio acerca do conhecimento produzido, aspectos que sinalizam para uma efetiva consolidação acadêmica da área no país.

\section{Notas}

(1) Curso com área de concentração em Comunicação e linha de pesquisa em Informação.

(2) Curso com área de concentração em Memória Social.

\section{Referencias}

Bertachini, M. L. (1996). O docente de biblioteconomia: construindo sua auto-imagem. // Informação \& Informação. Londrina. 1:1 (jan./jun. 1996) 41-44.

Brasil. Lei de Diretrizes e Bases da Educação Nacional. Lei n. 9.394, 20 de dezembro de 1996. Estabelece as diretrizes e bases da educação nacional. Disponível em: <http://www.pedagogiaemfoco.pro.br/193494-96.htm>. Acesso em: 25 mar. 2003.
Guimarães, J.A.C. (2001). Ensino de tratamento temático da Informação nos cursos de Biblioteconomia do Mercosul: uma análise histórica dos Encontros de Diretores e Docentes (1996-2000). // IV Encuentro de docentes de escuelas de bibliotecología y ciencia de la información del Mercosur. (Asunción, jul. 2001). Trabalho publicado em CD - ROM. 455-483.

Guimarães, J.A .C.; Miranda, F.; Santos, I.S. (2001). Ensino de tratamento temático da informação nos cursos de Biblioteconomia do Mercosul: análise e perspectivas de um core curriculum à luz dos avanços teóricos da área de organização do conhecimento. Marília. /Relatório de pesquisa apresentado do CNPq/.

Guimarães, J.A .C.; Danuelo, J.C.; Menezes, P.J. (2003). Ensino de tratamento temático da informação nos cursos de Biblioteconomia do Mercosul: uma análise de capacitação e produção científica docente com vistas ao delineamento de políticas integradas para área. Marília. Relatório de pesquisa apresentado do CNPq/.

Martins, G. M. (2002). Pós-graduação "stricto sensu" e capacitação docente no setor particular: panorama, dificuldades e perspectivas. http:// <www.abmes.org.br/ abmes/publica/revista/estud28/geraldo/geraldo.htm>. Acesso em: 27 set. 2002.

Morosini, M. C. (1999). Internacionalização de sistemas universitários: o Mercosul. // Nunes, B. F. Políticas e Estratégias de capacitação do corpo docente. Estudos e Debates. 20 (mar. 1999) 145-187.

Población, D. A. (2001). Produção científica: características das comunidades científicas brasileiras da área de Ciência de Informação segundo parâmetros cienciométricos. São Paulo: USP, 2001. Relatório de pesquisa apresentada ao CNPq.

Población, D.A. et al. (2007). A formação de pesquisadores na ártea de ciência da informação: docentes/doutores de cursos de pós-graduação. http://dci.ibict.br/archive/ 00000704/01/T043pdf. Acesso em 08.06.2007.

Puterman, J. (2000). A pós-graduação lato sensu no Brasil e sua implantação nas instituições isoladas particulares de ensino superior. Tese (doutorado em Educação) UnespMarília.

Souza, P. N. P. (2001). Estrutura e funcionamento do ensino superior brasileiro. São Paulo: Pioneira, 2001.

Smit, J. W. (2000). O profissional da informação e sua relação com as áreas de Biblioteconomia/Documentação, Arquivologia e Museologia. In: Valetim, M. P. (Org.) Profissionais da informação: formação, perfil e atuação profissional. São Paulo: Polis, 2000. 119-135.

Smit, J. W.; Dias, E. W.; Souza, R. F. de. (2002). Contribuição da Pós-graduação para a Ciência da Informação no Brasil: uma visão. DataGramaZero-Revista de Ciência da Informação. 3:6 (dez. 2002). http://www.dgz.org .br/dez02/Art04.htm

Sousa, M. R. Conselho de Educação do Ceará. Ofício n. 174/99-GAB, de 21 de junho de 1999. http://www.cec. ce.gov.br/cec-oficio1/4.htm. Acesso em: 28 set. 2002.

Souza, P. N. P. de. (1991). Estrutura e funcionamento do ensino superior brasileiro. São Paulo: Pioneira,1991.

Valentim, M. L. P.; Guimarães, J. A. C. (2002). Diretrizes políticas e estratégias para a formação docente voltadas à pesquisa e a extensão Brasil. // Transinformação. 14:1 (jan./jun.2002) 29-39. 